\title{
Practice Capacity to Address Patients' Social Needs and Physician Satisfaction and Perceived Quality of Care
}

Matthew S. Pantell, MD, MS ${ }^{1}$

Emilia De Marcbis, $M D^{2}$

Angeli Bueno, $M D^{2}$

Laura M. Gottlieb, MD, MPH ${ }^{2,3}$

'Department of Pediatrics, University of California, San Francisco, California

${ }^{2}$ Department of Family \& Community Medicine, University of California, San Francisco, California

${ }^{3}$ Social Interventions Research and Evaluations Network, University of California, San Francisco, California

Conflicts of interest: authors report none.

\section{CORRESPONDING AUTHOR}

Matthew S. Pantell, MD, MS

Department of Pediatrics

University of California, San Francisco

3333 California St, Ste 465

San Francisco, CA 94118

Matt.Pantell@ucsf.edu

\begin{abstract}
Recent studies have explored clinician impacts of health care-based interventions that respond to patients' social and economic needs. These studies were limited by available clinician data. We used the Commonwealth International Health Policy Survey of 890 primary care physicians to examine associations between clinic capacity to respond to patients' social needs and physician satisfaction, stress, and perceived medical care quality. Results suggest that perceived capacity to address social needs is strongly associated with both clinician satisfaction and perceived medical care quality. Our findings add to a growing literature on the potential return on investment of clinical interventions to address social needs.
\end{abstract}

Ann Fam Med 2019;17:42-45. https://doi.org/10.1370/afm.2334.

\section{INTRODUCTION}

$\Lambda$ growing body of evidence explores how addressing patients' adverse social circumstances in the context of health care delivery may affect their health and decrease avoidable health care cost and use. ${ }^{1}$ Two recent studies suggest that the impacts of greater clinical capacity to intervene on patients social and economic needs can extend beyond patients to clinicians, including a reduction in symptoms of burnout. ${ }^{2,3}$ These studies were limited in that they included few clinician-level variables. Using a large health policy study of primary care physicians, we explored associations between clinic capacity to address patients' social and economic needs and physician job satisfaction, stress, and perceived quality of medical care.

\section{METHODS}

Data came from the Commonwealth Fund's 2015 International Survey of Primary Care Physicians. ${ }^{4}$ in which questionnaires were distributed to a random sample of primary care physicians in 11 countries. This study included US physicians only. Initial recruitment was through mail ${ }_{i}$ questionnaires were self-completed on paper or online. A total of 1,001 US physicians responded (response rate $=30.9 \%$ ), of whom 170 had missing data on relevant variables. We used multiple imputation to impute missing data for 59 physicians, yielding a final analytic sample of 890 (11.1\%).

Two measures were used to capture capacity to address patient social needs. The first measure was preparedness to manage patients with social needs, defined as answering "well prepared" or "somewhat prepared" vs "not prepared" to the question, "How prepared is your practice to manage care for patients in need of social services in the community (eg, housing, meals, and transportation)?" The second measure was ease of care coordination, defined as answering "very easy" or "easy" vs "somewhat difficult" or "very difficult" to the question, "How easy or difficult is it to coordinate your patient's care with social services or other community providers when needed (eg, housing, meals, and transportation)?"

We assessed 6 physician outcomes: job satisfaction job stress $_{i}$ general income satisfaction, relative income satisfaction compared with special- 
ists; satisfaction with time spent with patients; and views on whether the quality of medical care has declined over the past 3 years.

All analyses were performed using Stata version 15.0 (StataCorp LP). To evaluate associations between perceived clinic capacity to address patient social needs and physician outcomes, we conducted bivariate and multivariate logistic regression analyses adjusting for demographic and practice variables. All models used survey weights to adjust for nonresponse based on known sociodemographic parameters of clinician sex, age, region, and specialty. Multiple imputation was performed to impute missing data for all variables except sex and outcomes. The study was considered exempt by our institutional review board.

\section{RESULTS}

Characteristics of the study sample are in Table 1. The largest share of the 890 physicians worked in a practice located in a city. Most reported often (36.6\%) or sometimes $(45.4 \%)$ caring for patients needing social services. On average, $33.7 \%$ felt that their clinic was prepared (well or somewhat) to manage patients needing these services, and $37.5 \%$ felt it was easy (very easy or easy) to coordinate patient care.

Physicians who reported practicing in a clinic prepared to manage patients with social needs had higher job satisfaction (adjusted odds ratio [aOR] for very satisfied vs very dissatisfied $=3.23 ; 95 \% \mathrm{CI}, 1.47-7.09)$, were more satisfied with amount of time spent with patients, (aOR for very satisfied vs very dissatisfied $=2.86 ; 95 \%$ CI, 1.37-6.00), and were more likely to think that the quality of medical care patients receive has improved $(\mathrm{aOR}=1.72 ; 95 \%$ CI, 1.19-2.49) (Table 2). Income satisfaction in general and relative to specialists was significantly associated with clinic preparedness to address patients with social needs initially, but not after controlling for how frequently the practice saw patients with social needs. There was no association with job stress.
Table 1. Physician and Practice Characteristics $(\mathbf{N}=890)$

\begin{tabular}{|c|c|c|c|}
\hline Characteristic & $\begin{array}{l}\text { Physicians, No., } \\
\text { Unweighted }\end{array}$ & $\begin{array}{l}\text { Physicians, \%, } \\
\text { Unweighted }\end{array}$ & $\begin{array}{l}\text { Physicians, \%, } \\
\text { Weighted }\end{array}$ \\
\hline \multicolumn{4}{|l|}{ Age, y } \\
\hline$<35$ & 45 & 5.1 & 5.7 \\
\hline $35-44$ & 192 & 21.6 & 20.1 \\
\hline $45-54$ & 244 & 27.4 & 29.4 \\
\hline $55-64$ & 296 & 33.3 & 29.3 \\
\hline$\geq 65$ & 110 & 12.4 & 15.5 \\
\hline Missing/Imputed & 3 & 0.3 & - \\
\hline \multicolumn{4}{|l|}{ Sex } \\
\hline Female & 345 & 38.8 & 39.1 \\
\hline Male & 545 & 61.2 & 60.9 \\
\hline \multicolumn{4}{|l|}{ Practice environment } \\
\hline City & 345 & 38.8 & 41.0 \\
\hline Suburb & 269 & 30.2 & 29.6 \\
\hline Small town & 167 & 18.8 & 18.6 \\
\hline Rural & 103 & 11.6 & 10.7 \\
\hline Missing/lmputed & 6 & 0.7 & - \\
\hline \multicolumn{4}{|c|}{ Year of graduation from residency } \\
\hline Before 1986 & 222 & 24.9 & 26.5 \\
\hline 1986-1995 & 229 & 25.7 & 25.0 \\
\hline $1996-2003$ & 221 & 24.8 & 25.9 \\
\hline 2004 or later & 206 & 23.1 & 22.6 \\
\hline Missing/Imputed & 12 & 1.3 & - \\
\hline \multicolumn{4}{|c|}{ Part of larger integrated provider system } \\
\hline No & 614 & 69.0 & 69.4 \\
\hline Yes & 273 & 30.7 & 30.6 \\
\hline Missing/Imputed & 3 & 0.3 & - \\
\hline \multicolumn{4}{|l|}{ US region } \\
\hline Northeast & 206 & 23.1 & 21.9 \\
\hline Midwest & 199 & 22.4 & 23.5 \\
\hline South & 290 & 32.6 & 33.0 \\
\hline West & 195 & 21.9 & 21.6 \\
\hline Missing/lmputed & 0 & 0.0 & - \\
\hline \multicolumn{4}{|l|}{ Medical specialty } \\
\hline $\begin{array}{l}\text { Family medicine/ } \\
\text { Medicine-pediatrics/ } \\
\text { General practice }\end{array}$ & 448 & 50.3 & 42.8 \\
\hline Internal medicine & 263 & 29.6 & 38.4 \\
\hline Pediatrics & 179 & 20.1 & 18.8 \\
\hline Missing/lmputed & 0 & 0.0 & - \\
\hline \multicolumn{4}{|c|}{ Size (full-time equivalent clinicians) } \\
\hline$\leq 1$ & 238 & 26.7 & 28.2 \\
\hline$>1$ to 3 & 242 & 27.2 & 26.7 \\
\hline$>3$ to 7 & 191 & 21.5 & 20.8 \\
\hline$>7$ & 208 & 23.4 & 24.3 \\
\hline Missing/lmputed & 11 & 1.2 & $\begin{array}{l}\text { - } \\
\text { continues }\end{array}$ \\
\hline
\end{tabular}

Physicians who reported that it was easy to coordinate patients' care with social services or other community clinicians had higher job satisfaction (aOR for very satisfied vs very dissatisfied $=2.75 ; 95 \% \mathrm{CI}, 1.33-5.67$ ), personal income satisfaction (aOR for very satisfied vs very dissatisfied $=2.28 ; 95 \% \mathrm{CI}, 1.22-4.26$ ), relative income satisfaction (aOR for very satisfied vs very dis- 
Table 1. Physician and Practice Characteristics $(\mathbf{N}=890)$ (continued)

\begin{tabular}{|c|c|c|c|}
\hline Characteristic & $\begin{array}{l}\text { Physicians, No., } \\
\text { Unweighted }\end{array}$ & $\begin{array}{l}\text { Physicians, \%, } \\
\text { Unweighted }\end{array}$ & $\begin{array}{l}\text { Physicians, \%, } \\
\text { Weighted }\end{array}$ \\
\hline \multicolumn{4}{|c|}{ Frequency of caring for patients needing social services } \\
\hline Often & 325 & 36.5 & 36.6 \\
\hline Sometimes & 399 & 44.8 & 45.4 \\
\hline Rarely & 140 & 15.7 & 15.2 \\
\hline Never & 23 & 2.6 & 2.7 \\
\hline Missing/Imputed & 3 & 0.3 & - \\
\hline \multicolumn{4}{|l|}{ Job satisfaction } \\
\hline Very satisfied & 156 & 17.5 & 18.1 \\
\hline Satisfied & 411 & 46.2 & 46.8 \\
\hline Somewhat dissatisfied & 264 & 29.7 & 29.0 \\
\hline Very dissatisfied & 55 & 6.2 & 6.0 \\
\hline Missing/lmputed & 4 & 0.4 & - \\
\hline \multicolumn{4}{|l|}{ Job stress } \\
\hline No stress & 94 & 10.6 & 10.9 \\
\hline Moderately stressed & 393 & 44.2 & 45.1 \\
\hline Very stressed & 282 & 31.7 & 31.2 \\
\hline Extremely stressed & 115 & 12.9 & 12.8 \\
\hline Missing/lmputed & 6 & 0.7 & - \\
\hline \multicolumn{4}{|l|}{ Satisfaction with income } \\
\hline Very satisfied & 137 & 15.4 & 15.5 \\
\hline Satisfied & 449 & 50.4 & 51.0 \\
\hline Somewhat dissatisfied & 209 & 23.5 & 23.2 \\
\hline Very dissatisfied & 90 & 10.1 & 10.3 \\
\hline Missing/Imputed & 5 & 0.6 & - \\
\hline \multicolumn{4}{|c|}{ Satisfaction with income relative to specialists } \\
\hline Very satisfied & 59 & 6.6 & 7.1 \\
\hline Satisfied & 187 & 21.0 & 22.0 \\
\hline Somewhat dissatisfied & 358 & 40.2 & 41.1 \\
\hline Very dissatisfied & 274 & 30.8 & 29.8 \\
\hline Missing/Imputed & 12 & 1.3 & - \\
\hline \multicolumn{4}{|c|}{ Satisfaction with amount of time spent with patients } \\
\hline Very satisfied & 69 & 7.8 & 8.5 \\
\hline Satisfied & 392 & 44.0 & 45.0 \\
\hline Somewhat dissatisfied & 330 & 37.1 & 36.7 \\
\hline Very dissatisfied & 92 & 10.3 & 9.8 \\
\hline Missing/Imputed & 7 & 0.8 & - \\
\hline \multicolumn{4}{|c|}{ Believes patient medical care quality is improving } \\
\hline No & 663 & 74.5 & 74.8 \\
\hline Yes & 225 & 25.3 & 25.2 \\
\hline Missing/Imputed & 2 & 0.2 & - \\
\hline \multicolumn{4}{|c|}{ Preparedness to manage patients in need of social services } \\
\hline $\begin{array}{l}\text { Well prepared/some- } \\
\text { what prepared }\end{array}$ & 293 & 32.9 & 33.7 \\
\hline Not prepared & 597 & 67.1 & 66.3 \\
\hline \multicolumn{4}{|l|}{ Ease of care coordination } \\
\hline Very easy/easy & 326 & 36.6 & 37.5 \\
\hline $\begin{array}{l}\text { Somewhat difficult/ } \\
\text { very difficult }\end{array}$ & 564 & 63.4 & 62.5 \\
\hline
\end{tabular}

care as recently improved $\left(\mathrm{aOR}=1.66_{\text {; }}\right.$ $95 \%$ CI $1.20-2.30$ ) in Table $2 \mathrm{a}$ and Supplemental Table $2 b$, available at http://www.AnnFamMed.org/content/17/1/42/suppl/DC1/. There was no significant association with job stress.

\section{DISCUSSION}

Clinic capacity to address patients' social needs was associated with higher physician job satisfaction and the perception that patient medical care has recently improved. Similarly, physicians reporting that care coordination (facilitating connection with social/ community resources) was easy were more likely to endorse higher job satisfaction. These findings suggest that the return on investment of activities related to patients' social and economic needs may extend beyond patient health and use of care to clinician satisfaction-closely tied with clinician burnout and retention. ${ }^{5-7}$ Health systems should consider clinician impacts when calculating costs and benefits of clinical team-based activities to respond to patients' social needs.

These data do not enable causality inferences; possibly, more satisfied physicians are more likely to believe that their clinics have the capacity to intervene on patients' social needs. The data are also self-reported by a small sample of US physicians, which may result in both selection and response bias, limiting generalizability. Finally, the data do not include information on time and efficiency burdens that may be associated with interventions around patients' social needs. ${ }^{8-10}$ Future work could link more objective measures of capacity to address social needs with other clinician outcomes.

To read or post commentaries in response to this article, see it online at http://www. AnnFamMed.org/content/17/1/42.

Key words: social needs; care coordination; vulnerable populations; acommunity/population satisfied $=3.08 ; 95 \% \mathrm{CI}, 1.67-5.67)$, and satisfaction with health; job satisfaction; quality of care; health policy; professional practice; disparities in health $\varepsilon$ health care; practice-based research; primary care

Submitted August 6, 2018; submitted, revised, October 3, 2018; accepted October 18, 2018. fied vs very dissatisfied $=3.39 .95 \% \mathrm{CI}, 1.63-7.06)$ and they were more likely to perceive the quality of medical

ANNALS OF FAMILY MEDICINE + WWW.ANNFAMMED.ORG + VOL. 17, NO.1 + JANUARY/FEBRUARY 2019 
Table 2a. Odds of Physician Outcomes Based on Practice Prepared to Address Patients' Social Needs $(\mathrm{N}=890)$

\begin{tabular}{|c|c|c|c|c|c|c|}
\hline \multirow[b]{3}{*}{ Outcome } & \multicolumn{6}{|c|}{ Practice is Well-Prepared to Address Patients With Social Needs } \\
\hline & \multicolumn{2}{|c|}{ Model 1} & \multicolumn{2}{|c|}{ Model 2} & \multicolumn{2}{|c|}{ Model 3} \\
\hline & OR $(95 \% \mathrm{Cl})$ & $P$ Value & OR $(95 \% \mathrm{Cl})$ & $P$ Value & OR $(95 \% \mathrm{Cl})$ & $P$ Value \\
\hline \multicolumn{7}{|l|}{ Job satisfaction } \\
\hline Very satisfied & $2.22(1.10-4.51)$ & .03 & $2.21(1.07-4.56)$ & .03 & $3.23(1.47-7.09)$ & .004 \\
\hline Satisfied & $1.44(0.74-2.78)$ & .28 & $1.43(0.73-2.82)$ & .30 & $2.05(0.99-4.25)$ & .053 \\
\hline Somewhat dissatisfied & $1.24(0.63-2.45)$ & .53 & $1.18(0.59-2.36)$ & .64 & $1.61(0.77-3.37)$ & .21 \\
\hline Very dissatisfied & Ref & - & Ref & - & Ref & - \\
\hline \multicolumn{7}{|l|}{ Job stress } \\
\hline No stress & $1.04(0.57-1.89)$ & .90 & $0.99(0.54-1.83)$ & .98 & $1.45(0.76-2.73)$ & .26 \\
\hline Moderately stressed & $0.81(0.52-1.29)$ & .38 & $0.82(0.51-1.30)$ & .40 & $1.02(0.62-1.70)$ & .93 \\
\hline Very stressed & $0.99(0.62-1.58)$ & .96 & $1.03(0.64-1.66)$ & .89 & $1.10(0.67-1.81)$ & .71 \\
\hline Extremely stressed & Ref & - & Ref & - & Ref & - \\
\hline \multicolumn{7}{|l|}{ Satisfaction with income } \\
\hline Very satisfied & $1.87(1.03-3.37)$ & .04 & $2.02(1.08-3.80)$ & .03 & $1.81(0.92-3.58)$ & .09 \\
\hline Satisfied & $1.32(0.78-2.21)$ & .30 & $1.38(0.80-2.39)$ & .25 & $1.38(0.78-2.44)$ & .27 \\
\hline Somewhat dissatisfied & $1.26(0.72-2.21)$ & .43 & $1.30(0.72-2.35)$ & .39 & $1.21(0.65-2.25)$ & .54 \\
\hline Very dissatisfied & Ref & - & Ref & - & Ref & - \\
\hline \multicolumn{7}{|l|}{ Satisfied with income relative to specialists } \\
\hline Very satisfied & $2.22(1.21-4.07)$ & .01 & $2.22(1.18-4.16)$ & .01 & $1.91(0.92-3.96)$ & .08 \\
\hline Satisfied & $1.13(0.74-1.72)$ & .57 & $1.08(0.70-1.66)$ & .73 & $1.03(0.64-1.66)$ & .89 \\
\hline Somewhat dissatisfied & $1.17(0.82-1.66)$ & .39 & $1.16(0.80-1.68)$ & .43 & $1.17(0.79-1.73)$ & .43 \\
\hline Very dissatisfied & Ref & - & Ref & - & Ref & - \\
\hline \multicolumn{7}{|c|}{ Satisfied with amount of time spent with patients } \\
\hline Very satisfied & $2.65(1.35-5.20)$ & .005 & $2.36(1.17-4.75)$ & .02 & $2.86(1.37-6.00)$ & .005 \\
\hline Satisfied & $1.06(0.64-1.76)$ & .82 & $0.98(0.58-1.64)$ & .93 & $1.34(0.77-2.34)$ & .30 \\
\hline Somewhat dissatisfied & $1.09(0.65-1.83)$ & .74 & $1.04(0.61-1.76)$ & .89 & $1.20(0.69-2.08)$ & .52 \\
\hline Very dissatisfied & Ref & - & Ref & - & Ref & - \\
\hline Patient medical care received is improving & $1.75(1.26-2.42)$ & .001 & $1.79(1.28-2.52)$ & .001 & $1.72(1.19-2.49)$ & .004 \\
\hline \multicolumn{7}{|l|}{$\mathrm{OR}=$ odds ratio; Ref $=$ reference group } \\
\hline $\begin{array}{l}\text { Notes: Using multiple imputation for all missing } \\
\text { pleted, specialty, clinic location, region of countr } \\
\text { covariates plus frequency practice sees patients }\end{array}$ & $\begin{array}{l}\text { except sex an } \\
\text { part of integra }\end{array}$ & e varia & $\begin{array}{l}\text { odel } 1 \text { covariate } \\
\text { ull-time equivale }\end{array}$ & $\begin{array}{l}\text { Model } \\
\text { ians in }\end{array}$ & $\begin{array}{l}\text { iates: age, sex, er } \\
\text { Model } 3 \text { covariat }\end{array}$ & $\begin{array}{l}\text { ining com- } \\
\text { model } 2\end{array}$ \\
\hline
\end{tabular}

Funding support: This article was supported by the Robert Wood Johnson Foundation (grant 74930).

Disclaimer: The contents of this article are solely the responsibility of the authors and do not represent the official views of the Robert Wood Johnson Foundation.

Supplemental Materials: Available at http://www.AnnFamMed. org/content/17/1/42/suppl/DC1/.

\section{References}

1. Gottlieb LM, Wing H, Adler NE. A systematic review of interventions on patients' social and economic needs. Am J Prev Med. 2017; 53(5):719-729.

2. Olayiwola JN, Willard-Grace R, Dubé K, et al. Higher perceived clinic capacity to address patients' social needs associated with lower burnout in primary care providers. J Health Care Poor Underserved. 2018;29(1):415-429

3. De Marchis EH, Knox M, Hessler D, et al. Perceived clinic capacity to address patients' social needs and family physician burnout. J Am Board Fam Med. 2019. In press.
4. The Commonwealth Fund. 2015 Commonwealth Fund international survey of primary care physicians in 10 nations. https://www. commonwealthfund.org/publications/surveys/2015/dec/2015commonwealth-fund-international-survey-primary-care-physicians. Published Dec 7, 2015. Accessed Jul 10, 2018.

5. Whitebird RR, Solberg LI, Crain AL, et al. Clinician burnout and satisfaction with resources in caring for complex patients. Gen Hosp Psychiatry. 2017;44:91-95.

6. Bodenheimer T, Sinsky C. From triple to quadruple aim: care of the patient requires care of the provider. Ann Fam Med. 2014;12(6): 573-576.

7. Shanafelt T, Goh J, Sinsky C. The business case for investing in physician well-being. JAMA Intern Med. 2017;177(12):1826-1832.

8. Hayashi AS, Selia E, McDonnell K. Stress and provider retention in underserved communities. J Health Care Poor Underserved. 2009; 20(3):597-604.

9. VITAL WorkLife, Inc. VITAL WorkLife and Cejka Search Physician Stress and Burnout Survey. http://www. physicianwellnessservices. com/news/stresssurvey.php. Accessed Jul 5, 2018.

10. Spinelli WM, Fernstrom KM, Britt H, Pratt R. "Seeing the patient is the joy:" a focus group analysis of burnout in outpatient providers. Fam Med. 2016;48(4):273-278. 